\title{
Prescrizione e valutazione nel tempo della dose dialitica con test di equilibrio specifico per la dialisi peritoneale Tidal
}

\author{
A. Edefonti ${ }^{1}$, M. Picca ${ }^{1}$, G. Consalvo ${ }^{1}$, L. Ghio ${ }^{1}$, M. Giani ${ }^{1}$, B. Damiani ${ }^{1}$, \\ A. Dal $\mathrm{Col}^{2}$, R. Galato ${ }^{2}$ \\ ${ }^{1}$ Clinica Pediatrica II, Università di Milano \\ ${ }^{2}$ Divisione di Nefrologia, Ospedale Niguarda, Milano
}

a prescrizione e la valutazione nel tempo della dose dialitica sono diventate una parte importante del trattamento di pazienti in dialisi peritoneale. Il metodo "gold standard" per valutare la dialisi peritoneale rimane la misurazione delle clearance dei soluti, ma la raccolta di grandi volumi di dialisato può essere origine di inconvenienti e di inesattezze, soprattutto per i pazienti in dialisi peritoneale automatizzata (APD) (1). Il test di equilibrio peritoneale (PET), in origine introdotto per valutare le caratteristiche di permeabilità della membrana peritoneale (2), è stato anche utilizzato per estrapolare le clearance giornaliere dei piccoli soluti in pazienti in CAPD e NIPD/ CCPD $(1,3)$, ma rimane tuttora controverso se il PET sia in grado o meno di predire con accuratezza le clearance dei soluti misurate con i metodi di raccolta tradizionali (4-7). Recentemente il nostro gruppo ha studiato questo problema in pazienti trattati con dialisi peritomeale Tidal (TPD) (9), che rappresenta una modalità promettente di trattamento dell'uremia terminale (8). È stato da noi ipotizzato che la possibilità di predire con accuratezza le clearance peritoneali dei soluti sia più elevata utilizzando i rapporti dialisato/plasma (D/P) derivati da un test di equilibrio specifico per la TPD (TPD test), piuttosto che $\mathrm{i}$ rapporti $\mathrm{D} / \mathrm{P}$ ottenuti da PET standard, mentre non sono state valutate le correlazioni tra le clearance stimate dai vari rapporti $\mathrm{D} / \mathrm{P}$ e le clearance misurate nonché la precisione statistica della predizione. In realtà, manca tuttora una precisa dimostrazione dell'attendibilità del TPD test nel valutare la clearance peritoneale dei soluti. L'obiettivo del nostro studio è stato quello di determinare: 1) se i rapporti D/P per creatinina ed urea, calcolati a vari tempi del PET, correlino o meno con le clearance peritoneali misurate dei soluti; 2) se i rapporti di $\mathrm{D} / \mathrm{P}$ per creatinina ed urea ottenuti da test di equilibrio specifico per TPD siano correlati o meno con le clearance peritoneali misurate dei soluti; 3) la precisione statistica delle clearance peritoneali della creatinina e dell'urea derivate dal test di equilibrio TPD nel predire le clearance reali ottenute in $\mathrm{TPD} ; 4)$ le relazioni tra i rapporti $\mathrm{D} / \mathrm{P}$ derivati dal PET e quelli derivati dal TPD test, allo scopo di accertare se questi ultimi forniscano informazioni anche sulla permeabilità peritoneale.

\section{Pazienti}

Sono stati studiati 11 pazienti con IR terminale di età media pari a $12.4 \pm 3.3$ anni e peso medio di $34.2 \pm 15 \mathrm{~kg}$, in condizioni cliniche stabili e trattati con TPD per $13.8 \pm 6.5$ mesi. Le apparecchiature utilizzate sono state le Gambro PD100 (Gambro, Lund, Svezia). La prescrizione dialitica in TPD prevedeva flussi di dialisi di $65 \pm 14 \mathrm{ml} / \mathrm{kg} / \mathrm{h}$ con un volume di scambio Tidal medio (pari al 50\% del volume di riempimento) di $620 \pm 198 \mathrm{ml}$ (range 300-1000), un tempo medio di stasi di $11.8 \pm 2.5$ minuti, un numero medio di cicli pari a $29.8 \pm 6.1$ ed una durata della seduta pari a $9.7 \pm 0.41$ ore.

\section{Metodologia clinica e di laboratorio}

Tutti i pazienti sono stati dapprima sottoposti al test specifico di equilibrio per la TPD (TPD test), consistente in un trattamento domiciliare standardizzato di dialisi peritoneale Tidal con volume di riempimento pari a $40 \mathrm{ml} / \mathrm{kg}$ e soluzione di glucosio $1.5 \%$, volumi di scambio pari al $50 \%$ del riempimento, tempo di stasi 
di 10 minuti e durata del trattamento di 8 ore. I volumi di drenaggio Tidal del $5^{\circ} \mathrm{e}$ $7^{\circ}$ scambio, considerati, in base a dati di cinetica, il punto di equilibrio della dialisi Tidal, sono stati raccolti dai genitori dei pazienti, così come il volume totale del dialisato al termine del trattamento Tidal, allo scopo di effettuare la misurazione della creatinina, urea e glucosio. La creatinina del dialisato è stata corretta per l'interferenza del glucosio. Un campione di sangue è stato prelevato nel servizio di dialisi due ore dopo la fine del trattamento con TPD per la determinazione della creatinina e dell'urea. Questi livelli di creatinina ed urea sierica sono stati considerati approssimare i valori ottenibili a metà del trattamento dialitico (10). Ciascun paziente è stato quindi sottoposto a PET standardizzato secondo Twardowski (2), modificato per l'età pediatrica (11) e interrotto dopo 120 minuti (12), per ottenere una durata del test paragonabile a quella del TPD test. Il volume di riempimento era di $40 \mathrm{ml} / \mathrm{kg}$. Il dialisato è stato raccolto al tempo 0 e dopo $15,30,60$ e 120 minuti; i campioni di siero sono stati prelevati a 120 minuti.

\section{Calcoli}

I rapporti $\mathrm{D} / \mathrm{P}$ per creatinina ed urea so- no stati calcolati al $5^{\circ}$ e al $7^{\circ}$ scambio Tidal, sul volume totale del dialisato Tidal, e a $0,15,30,60,120$ minuti del PET. Le clearance dialitiche di creatinina ed urea sono state calcolate secondo la formula standard (D/P $\times$ V) e rapportate alla superficie corporea. Le clearance sono state ottenute secondo 2 modalità: 1) dal reale valore misurato sul volume totale del dialisato (clearance misurate); 2) dai valori $\mathrm{D} / \mathrm{P}$ derivati dalla raccolta di dialisato al $5^{\circ}$ e al $7^{\circ}$ scambio TPD (clearance stimate).

\section{Analisi dei dati}

Tutti i dati sono stati introdotti in un programma StatView. Medie, deviazioni standard e coefficienti di correlazione sono stati calcolati con tale programma. Valori di $\mathrm{p}<0.05$ sono stati considerati significativi. I valori stimati di clearance dialitiche sono stati confrontati con quelli delle clearance dialitiche misurate. Un errore superiore al $10 \%$ è stato considerato clinicamente significativo (6).

\section{Risultati}

In Tabella I sono riportate le relazioni tra $\mathrm{i}$ rapporti $\mathrm{D} / \mathrm{P}$ per creatinina e urea a
15, 30, 60 e 120 minuti del PET e sul volume totale del dialisato TPD e le clearance dialitiche misurate di creatinina e urea. I coefficienti di correlazione sono più elevati per i tempi di PET più lunghi che per quelli brevi, per $\mathrm{i}$ rapporti $\mathrm{D} / \mathrm{P}$ derivati dal dialisato totale TPD rispetto alle clearance dialitiche misurate e per le variabili della creatinina rispetto a quelle dell'urea. Correlazioni particolarmente significative sono state osservate tra $\mathrm{i}$ rapporti $\mathrm{D} / \mathrm{P}$ per creatinina ed urea derivati dal dialisato del $5^{\circ}$ e del $7^{\circ}$ scambio e i rapporti D/P derivati dal volume totale del dialisato TPD. Sulla base delle equazioni riportate in Figura 1 e 2 abbiamo calcolato il rapporto $\mathrm{D} / \mathrm{P}$ per creatinina e urea su tutta la seduta Tidal dai rapporti $\mathrm{D} / \mathrm{P}$ del $5^{\circ}$ e del $7^{\circ}$ scambio. Le clearance dialitiche possono così essere calcolate moltiplicando i valori di D/P ottenuti dalle equazioni per il volume del dialisato. Le clearance dialitiche stimate sono risultate significativamente correlate con le clearance misurate nel corso della seduta Tidal standardizzata (Figg. 3 e 4). Abbiamo inoltre confrontato le clearance dialitiche stimate con quelle misurate. La precisione del metodo è stata valutata determinando la percentuale di sovrastima e sottostima oltre il $10 \%$ delle variabili sopra citate (Tab. II).

TABELLA I - CORRELAZIONI TRA I RAPPORTI D/P PER CREATININA E UREA A 15, 30, 60 E 120 MINUTI DEL PET E I RAPPORTI D/P PER CREATININA E UREA SUL VOLUME TOTALE DEL DIALISATO TPD (TOTAL TPD) E LE CLEARANCE DIALITICHE MISURATE DI CREATININA ED UREA (L/giorno/1.73 m²)

\begin{tabular}{|c|c|c|c|c|c|}
\hline \multicolumn{2}{|l|}{$\mathrm{D} / \mathrm{P}$ creatinina } & 15 min PET & 30 min PET & $60 \mathrm{~min}$ PET & 120 min PET \\
\hline Total TPD & $0.27 \pm 0.10$ & $\begin{array}{l}r=0.58 \\
p=0.06\end{array}$ & $\begin{array}{l}r=0.69 \\
p=0.01\end{array}$ & $\begin{array}{c}r=0.78 \\
p=0.004\end{array}$ & $\begin{array}{c}\mathrm{r}=0.87 \\
\mathrm{p}=0.0004\end{array}$ \\
\hline Cl. peritoneale & $7.43 \pm 3.16$ & $\begin{array}{c}r=0.44 \\
p=0.1\end{array}$ & $\begin{array}{l}r=0.62 \\
p=0.03\end{array}$ & $\begin{array}{l}r=0.69 \\
p=0.01\end{array}$ & $\begin{array}{c}r=0.75 \\
p=0.007\end{array}$ \\
\hline \multirow[t]{2}{*}{ D/P urea } & & 15 min PET & $30 \mathrm{~min}$ PET & $60 \mathrm{~min}$ PET & 120 min PET \\
\hline & & $0.31 \pm 0.07$ & $0.45 \pm 0.08$ & $0.61 \pm 0.10$ & $0.79 \pm 0.11$ \\
\hline Total TPD & $0.40 \pm 0.12$ & $\begin{array}{c}r=0.40 \\
p=0.2\end{array}$ & $\begin{array}{l}r=0.58 \\
p=0.06\end{array}$ & $\begin{array}{l}r=0.60 \\
p=0.06\end{array}$ & $\begin{array}{c}r=0.81 \\
p=0.002\end{array}$ \\
\hline Cl. peritoneale & $10.24 \pm 3.91$ & $\begin{array}{c}r=0.18 \\
p=0.6\end{array}$ & $\begin{array}{c}r=0.50 \\
p=0.1\end{array}$ & $\begin{array}{c}r=0.45 \\
p=0.1\end{array}$ & $\begin{array}{l}r=0.60 \\
p=0.06\end{array}$ \\
\hline
\end{tabular}


Fig. 1 - Correlazioni tra $i$ rapporti $D / P$ per creatinina al $5^{\circ}$ e $7^{\circ}$ scambio TPD (5th TPD exch D/P, 7th $T P D$ exch $D / P)$ e il rapporto D/P per creatinina sul volume totale del dialisato $T P D($ total TPD D/P).
Fig. 2 - Correlazioni tra $i$ rapporti $D / P$ per urea al $5^{\circ}$ e $7^{\circ}$ scambio TPD $(5$ th TPD exch $D / P, 7$ th $T P D$ exch $D / P)$ e il rapporto $D / P$ per urea sul volume totale del dialisato TPD (total TPD $D / P)$.
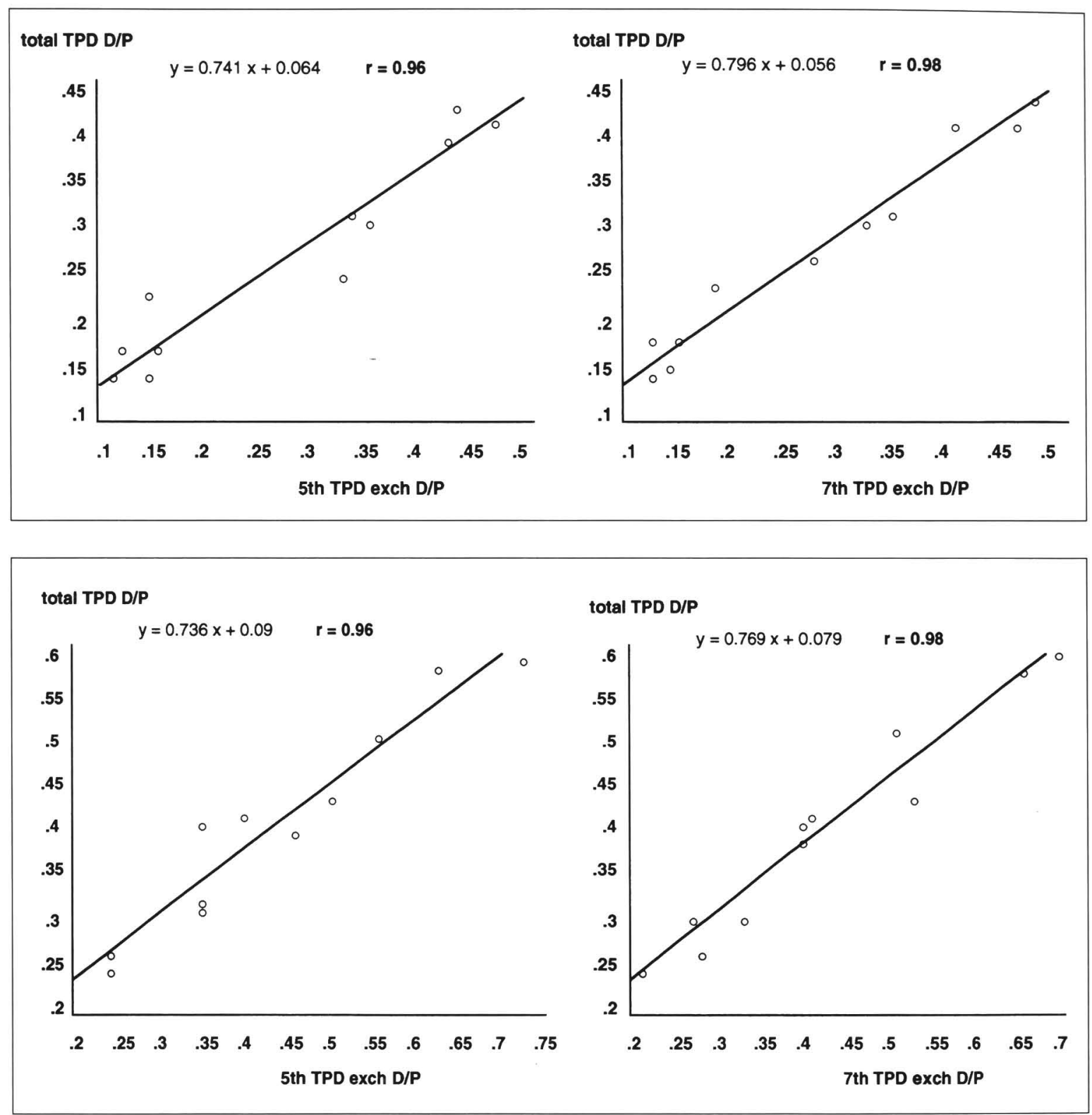

TABELLA II - ATTENDIBILITÀ DELLE CLEARANCE PERITONEALI STIMATE DI CREATININA E UREA

\begin{tabular}{|c|c|}
\hline Metodo & $\begin{array}{c}\text { Sovrastima } \\
>10 \%\end{array}$ \\
\hline
\end{tabular}

\section{Creatinina}

derivata dal $5^{\circ}$ scambio

$\begin{array}{cl}18.2 \% & 9.1 \% \\ 0 \% & 9.1 \%\end{array}$

derivata dal $7^{\circ}$ scambio

$9.1 \%$

\section{Urea}

derivata dal $5^{\circ}$ scambio

$\begin{array}{cc}9.1 \% & 9.1 \% \\ 9.1 \% & 0 \%\end{array}$

derivata dal $7^{\circ}$ scambio
Le clearance stimate derivate dal $5^{\circ}$ scambio erano entro il $10 \%$ dei valori misurati nel $72.7 \%$ dei pazienti per la creatinina e nell' $81.8 \%$ dei pazienti per l'urea. Le clearance stimate derivate dal $7^{\circ}$ scambio erano entro il $10 \%$ dei valori misurati nel $90.9 \%$ dei pazienti, sia per la creatinina che per l'urea. L'analisi delle relazioni tra i rapporti $\mathrm{D} / \mathrm{P}$ per creatinina ed urea al $5^{\circ}$ e al $7^{\circ}$ scambio TPD e i rapporti D/P per creatinina ed urea derivati da differenti tempi di PET, ha dimostrato più elevati coefficienti di correlazione per i tempi di PET più lunghi rispetto a quelli brevi e per le variabili basate sulla creatinina rispetto a quelle basate sull'urea (Tab. III). 


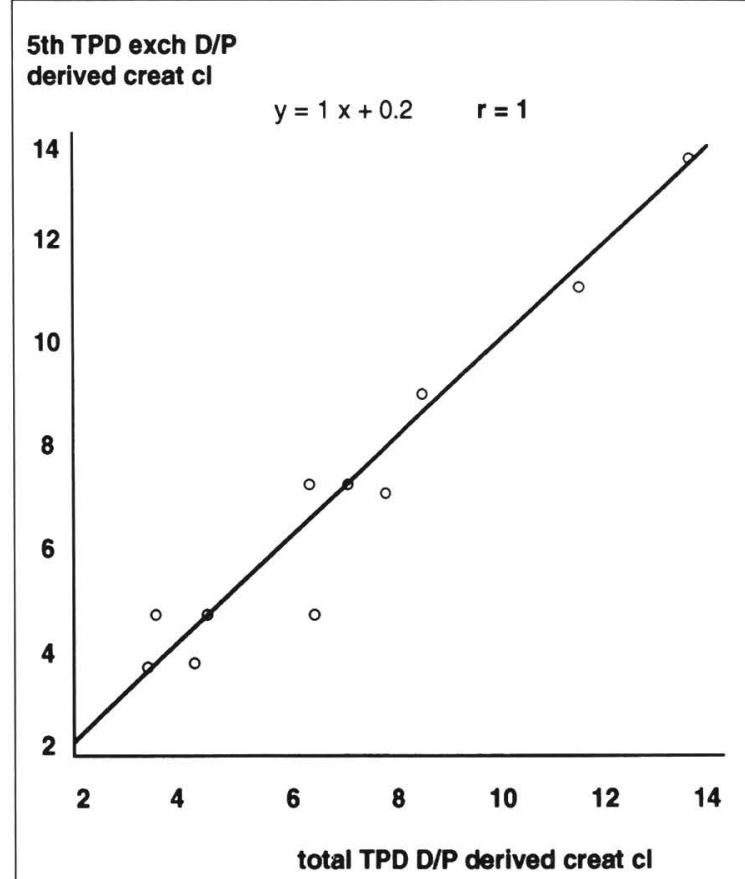

\section{7th TPD exch D/P \\ derived creat $\mathrm{Cl}$}

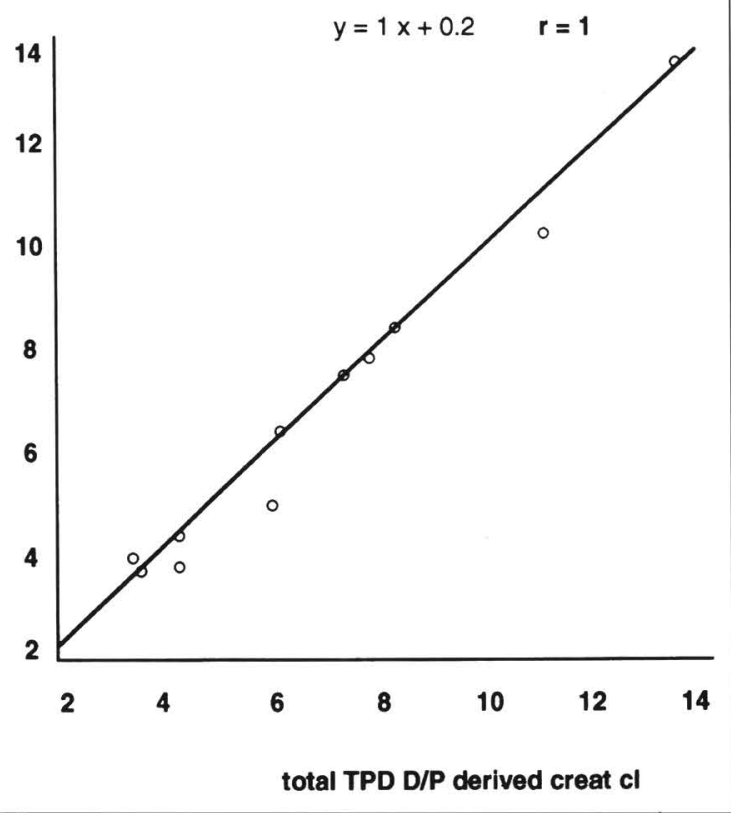

Fig. 3 - Correlazioni tra clearance dialitiche della creatinina derivate dai rapporti $D / P$ del $5^{\circ}$ (5th TPD exch $D / P$ derived (reat cl) $e$ del $7^{\circ}$ scambio TPD (7th TPD exch D/P derived (reat cl) e la clearance dialitica della creatinina misurata sul volume totale del dialisato TPD (total TPD D/P derived (reat ( $)$. Tutti $i$ valori sono espressi in L/giornol $1.73 \mathrm{~m}^{2}$.

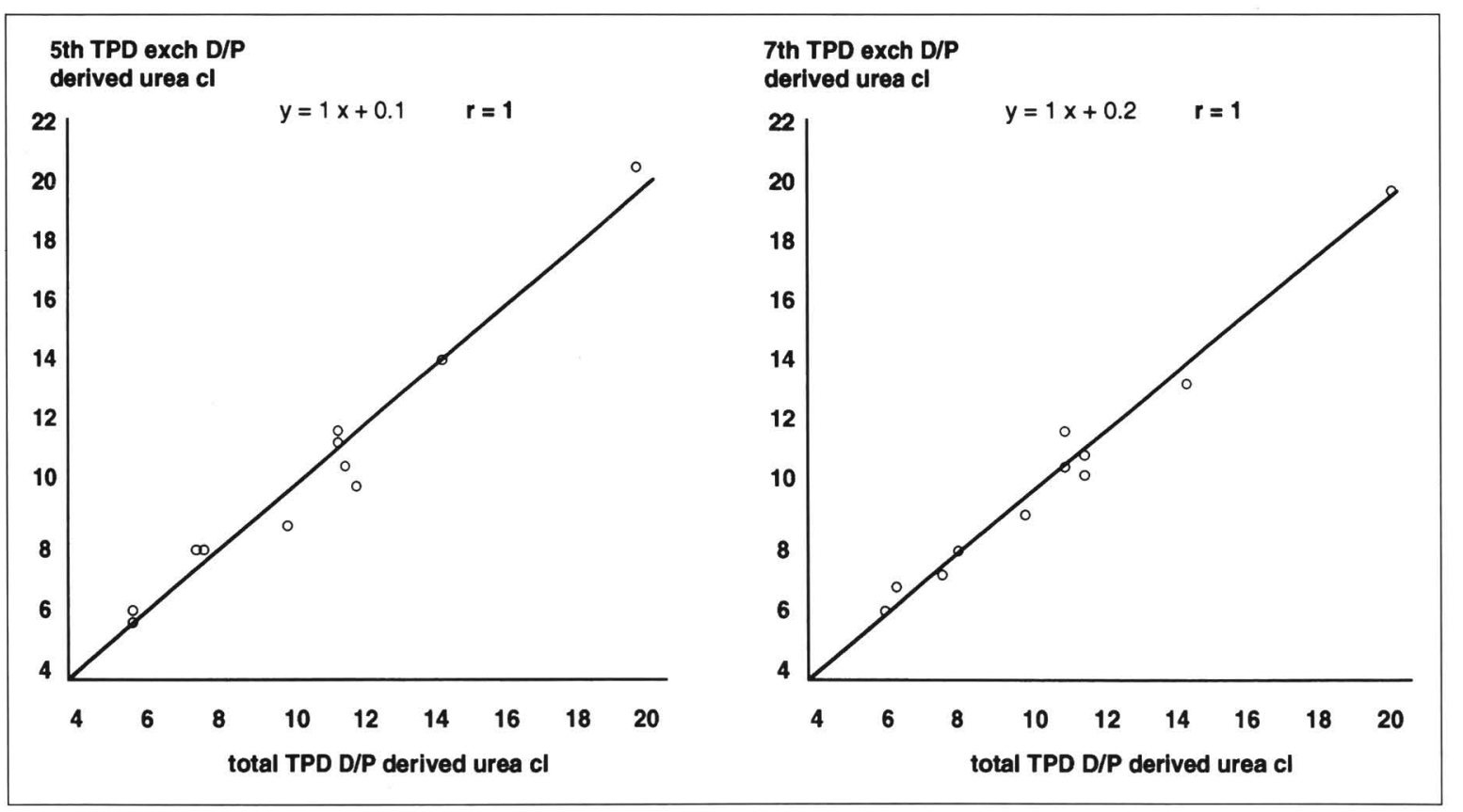

Fig. 4 - Correlazioni tra clearance dialitiche dell' $u$ rea derivate dai rapporti $D / P$ del $5^{\circ}$ (5th TPD exch $D / P$ derived urea $\mathrm{cl}$ ) e del $7^{\circ}$ scambio TPD (7th TPD exch $D / P$ derived urea $c l) e$ la clearance dialitica dell'urea misurata sul volume totale del dialisato TPD (total TPD D/P derived urea cl). Tutti i valori sono espressi in L/giorno/1.73 $m^{2}$.

\section{Discussione}

È tuttora dibattuto se il PET test predica con accuratezza, nei pazienti trattati con APD, le clearance di creatinina ed urea ottenute da studi di clearance tradizionali. Secondo Wolf (1) e Mendley (5), sia negli adulti che nei bambini trattati con NPD o CCPD, i rapporti D/P per creatinina ed urea, determinati con i metodi di clearance sono correlati con quelli otte- nuti con il PET test. Burkart (6), invece, ha trovato che i valori di clearance dialitica derivati dal PET sia sovrastimano che sottostimano la clearance misurata delle 24 ore. Commentando un articolo di Mendley, Morgenstern (4) ha osservato che i valori calcolati derivati dal PET e i valori corrispondenti misurati dal dialisato raccolto sulle 24 ore non sono identici e che il test di equilibrio dovrebbe essere considerato "vicino alla realtà ma non completamente esatto". Non è stato pubblicato nessuno studio che abbia applicato i dati derivati dal PET alla dialisi Tidal, il che ci ha spinto ad affrontare il problema. Si poteva presumere che $\mathrm{i}$ rapporti $\mathrm{D} / \mathrm{P}$ per creatinina ed urea calcolati ai tempi brevi del PET (15 e 30 minuti), più vicini agli usuali brevi tempi di sosta Tidal, possano predire la clearance misurata in modo più attendibile (1). Nel nostro studio, invece, i rapporti $\mathrm{D} / \mathrm{P}$ calcolati ai tempi brevi del PET sono risultati meno significativa- 
TABELLA III - CORRELAZIONI TRA I RAPPORTI D/P PER CREATININA E UREA DERIVATI A 15, 30, 60 E 120 MINUTI DEL PET E I RAPPORTI D/P PER CREATININA E UREA DERIVATI DAL $5^{\circ}$ E $7^{\circ}$ SCAMBIO TPD (5th TPD exch, 7th TPD exch)

\begin{tabular}{l|cccc}
\hline \multirow{2}{*}{ D/P creatinina } & 15 min PET & 30 min PET & 60 min PET & 120 min PET \\
& $0.23 \pm 0.08$ & $0.33 \pm 0.10$ & $0.47 \pm 0.14$ & $0.65 \pm 0.17$ \\
\cline { 2 - 5 } 5th TPD exch $0.28 \pm 0.14$ & $\mathrm{r}=0.70$ & $\mathrm{r}=0.81$ & $\mathrm{r}=0.88$ & $\mathrm{r}=0.93$ \\
& $\mathrm{p}=0.01$ & $\mathrm{p}=0.002$ & $\mathrm{p}=0.0003$ & $\mathrm{r}=0.89$ \\
7th TPD exch $0.27 \pm 0.13$ & $\mathrm{r}=0.61$ & $\mathrm{r}=0.74$ & $\mathrm{r}=0.80$ & $\mathrm{p}=0.0002$ \\
& $\mathrm{p}=0.04$ & $\mathrm{p}=0.008$ & $\mathrm{p}=0.002$ & 120 min PET \\
& 15 min PET & 30 min PET & 60 min PET & $0.79 \pm 0.11$ \\
\hline \multirow{2}{*}{ D/P urea } & $0.31 \pm 0.07$ & $0.45 \pm 0.08$ & $0.61 \pm 0.10$ & $\mathrm{r}=0.76$ \\
& $\mathrm{r}=0.42$ & $\mathrm{r}=0.43$ & $\mathrm{r}=0.62$ & $\mathrm{p}=0.01$ \\
& $\mathrm{p}=0.2$ & $\mathrm{p}=0.1$ & $\mathrm{p}=0.03$ & $\mathrm{r}=0.78$ \\
5th TPD exch $0.42 \pm 0.15$ & $\mathrm{r}=0.36$ & $\mathrm{r}=0.52$ & $\mathrm{p}=0.54$ & $\mathrm{p}=0.007$
\end{tabular}

mente correlati con i rapporti $\mathrm{D} / \mathrm{P}$ derivati dal dialisato totale Tidal rispetto ai rapporti $\mathrm{D} / \mathrm{P}$ calcolati ai tempi più lunghi del PET (60 e 120 minuti). Una possibile spiegazione è che i valori dei rapporti D/P per creatinina e urea ai tempi del PET antecedenti ai 120 minuti siano ancora lontani dall'equilibrio, mentre i valori del rapporto $\mathrm{D} / \mathrm{P}$ al $5^{\circ}$ e al $7^{\circ}$ scambio della seduta Tidal corrispondono già al valore del rapporto $\mathrm{D} / \mathrm{P}$ derivato dal volume totale Tidal. La correlazione tra $\mathrm{i}$ rapporti $\mathrm{D} / \mathrm{P}$ derivati ai tempi 60 e 120 minuti del PET e la clearance dialitica misurata in TPD è risultata più significativa per la creatinina che per l'urea, benché il livello di significatività non sia stato alto in entrambi i casi. Ci sono, infatti differenze nelle modalità e nei tempi di equilibrio tra il PET test e il TPD test (metà del dialisato scambiato per ogni ciclo ed un più rapido equilibrio dei soluti in TPD), così come tra creatinina $\mathrm{e}$ urea, che potrebbero spiegare i risultati osservati. Di conseguenza, la predizione della clearance peritoneale dei soluti in TPD sulla base del rapporto $\mathrm{D} / \mathrm{P}$ derivato dai tempi 60 e 120 minuti del PET non appare sufficientemente precisa. Peraltro, abbiamo osservato una correlazione altamente significativa tra $\mathrm{i}$ rapporti $\mathrm{D} / \mathrm{P}$ per creatinina ed urea derivati dal $5^{\circ} \mathrm{e}$ dal $7^{\circ}$ scambio Tidal di una seduta TPD standardizzata e i rapporti $\mathrm{D} / \mathrm{P}$ derivati dal volume totale del dialisato Tidal. Una correlazione significativa è stata anche ritrovata tra le clearance dialitiche misurate e stimate di creatinina ed urea della stessa seduta. In particolare la percentuale di valori di clearance dialitica stimati dal TPD test che sovra o sottostima del $10 \%$ le clearance misurate è più bassa di quella riportata per il PET (6). Inoltre, i valori derivati dal $7^{\circ}$ scambio Tidal sono lievemente più accurati dei valori derivati dal $5^{\circ}$ scambio. Nel complesso, le variabili derivate dal TPD test sono più attendibili rispetto a quelle derivate dal PET test nel predire le clearance dei soluti con basso peso molecolare. Una possibile spiegazione di questi risultati è che il rapporto D/P per creatinina ed urea durante una seduta TPD standardizzata si stabilizza progressivamente dal $5^{\circ} \mathrm{scam}$ bio in avanti; inoltre, i volumi di riempimento e di scambio e la concentrazione di glucosio usati durante il TPD test non differiscono da quelli usati durante una normale seduta TPD, il che riduce la discrepanza tra clearance attese e reali riportate in letteratura per il PET test (4, 6). Quindi, conoscendo il rapporto $\mathrm{D} / \mathrm{P}$ per creatinina e urea derivato da ogni scambio successivo al $5^{\circ}$ (e preferibilmente al $7^{\circ}$ ), di una seduta Tidal standardizzata e il volume di dialisato che viene scambiato, è possibile prevedere le clearance reali in corso di dialisi Tidal utilizzando la formula della clearance $(\mathrm{D} / \mathrm{P} \mathrm{x}$ V) e, conseguentemente, predire l'indicazione alla TPD di un dato paziente. Sebbene molti Autori (7) convengano che la misurazione delle clearance peritoneali a partire da tutto il dialisato raccolto è il metodo più preciso da utilizzare per la prescrizione e valutazione nel tempo della dose dialitica, una possibilità di errore può nascere anche in questo caso dalla scarsa compliance dei genitori o dei pazienti e dalla non corretta raccolta e miscelazione del dialisato $(1,5)$. La raccolta del dialisato da uno scambio dopo il $5^{\circ}$ di una seduta TPD standardizzata è facile da eseguire, richiede poco tempo, e permette una sostanziale riduzione dei problemi ed errori che possono accompagnare la raccolta di grandi volumi di dialisato (1). Inoltre, in confronto al PET, il test di equilibrio specifico per la TPD è meno complicato, fa risparmiare tempo agli infermieri, riduce il rischio teorico di peritoniti correlato al PET (4) e può quindi essere utilizzato più facilmente nella pratica clinica. Nel nostro studio abbiamo anche osservato una significativa correlazione tra i tempi $60 \mathrm{e}$ 120 minuti del PET e il rapporto D/P per creatinina derivato dal $5^{\circ}$ e dal $7^{\circ}$ scambio Tidal. È noto che le proprietà di tra- 
sporto della membrana peritoneale possono essere classificate sulla base del rapporto $\mathrm{D} / \mathrm{P}$ per creatinina determinato con il PET standard, e che dei valori di riferimento sono disponibili anche per l'età pediatrica (13-16). Il riscontro della correlazione sopra citata suggerisce che il nuovo TPD test da noi proposto possa essere usato per ottenere informazioni sulle caratteristiche di permeabilità della membrana peritoneale nei pazienti trattati con dialisi Tidal. In conclusione, la predizione delle clearance dei soluti di basso peso molecolare basata sul rapporto $\mathrm{D} / \mathrm{P}$ derivato dal PET è, in corso di TPD, meno accurata di quella basata sul $\mathrm{D} / \mathrm{P}$ derivato dalla raccolta di dialisato al $5^{\circ}$ e $7^{\circ}$ scambio Tidal. Una stima della eventuale indicazione alla dialisi Tidal e la valutazione routinaria della dose di dialisi Tidal fornita possono essere attuate sulla base della clearance predetta (calcolata dai rapporti $\mathrm{D} / \mathrm{P}$ per creatinina ed urea derivati da ogni scambio dopo il $5^{\circ}$, e preferibilmente dopo il $7^{\circ}$, di una seduta standardizzata TPD) e del volume di dialisato che deve essere scambiato per seduta al fine di realizzare una adeguata prescrizione dialitica. Questo metodo appare accurato per la pratica clini$\mathrm{ca}$, ma non può essere ancora raccomandato per scopi di ricerca essendo stato valutato solo su un piccolo numero di pazienti.

\section{BIBLIOGRAFIA}

1.

Wolf CJ, Polsky J, Ntoso KA, Koethe JD, Gerhardt RE. Adequacy of dialysis in CAPD and cycler PD; the PET is enough. In: Khanna R, Nolph KD, Prowant BF, Twardowski ZJ, Oreopoulos DG, eds. Adv Perit Dial 1992; 8: 208-11.

2. Twardowski ZJ, Nolph KD, Khanna R, et al. Peritoneal equilibration test. Perit Dial Bull 1987; 7: 138-47.

3. Blake PG, Sombolos K, Abraham G, et al. Lack of correlation between urea kinetic indices and clinical outcomes in CAPD patients. Kidney Int 1991; 39: 700-6.

4. Morgenstern BZ. Equilibration testing: close, but not quite right. Pediatr Nephrol 1993; 7: 290-1.

5. Mendley SR, Umans JG, Majkowski NL. Measurement of peritoneal dialysis delivery in children. Pediatr Nephrol 1993; 7: 284-9.

6. Burkart JM, Jordan JR, Rocco MV. Assessment of dialysis dose by measured clearance versus extrapolated data. Perit Dial Int 1993; 13: 184-8.

7.

Busch S, Schreiber M, Bodnar $\mathrm{D}$, et al. The 24-hour $\mathrm{D} / \mathrm{P}$ ratio is a convenient screen for identifying altered peritoneal transport rates. In: Khanna R, Nolph KD, Prowant BF, Twardowski ZJ, Oreopoulos DG, eds. Adv Perit Dial 1993; 9: 119-23.

8. Flanigan M, Pflederer T, Doyle $\mathrm{C}$, et al. Tidal peritoneal dialysis in children: initial experiences. Dial Transplant 1993; 9: 555-62.

9. Edefonti A, Picca M, Consalvo $\mathrm{G}$, et al. A new test for tidal peritoneal dialysis prescription. Adv Perit Dial 1994; 10: 112-5.

10. Nolph KD, Twardowski ZJ, Keshaviah PR. Weekly clearances of urea and creatinine on CAPD and
NIPD. Perit Dial Int 1992; 12: 298303.

11. Edefonti A, Picca M, Galato $\mathrm{R}$, et al. Evaluation of the peritoneal equilibration test in children on chronic peritoneal dialysis. Perit Dial Int 1993; 13 (suppl 2) : 260-2.

12. Zabetakis PM, Krapf R, De Vita MV, Gleim GW, Michelis MF. Determining peritoneal dialysis prescriptions by employing a patientspecific protocol. Perit Dial Int 1993; 13: 189-93.

13. Ellis EN, Watts K, Wells TG, Arnold WC. Use of peritoneal equilibration test in pediatric dialysis patients. In: Khanna R, Nolph KD, Prowant BF, Twardowski ZJ, Oreopoulos DG, eds. Adv Perit Dial 1991; 7: 259-61.

14. Schaefer F, Langenbeck D, Heckert KH, Schärer K, Mehls O. Evaluation of peritoneal solute transfer by the peritoneal equilibration test in children. In: Khanna R, Nolph KD, Prowant BF, Twardowski ZJ, Oreopulos DG, eds. Adv Perit Dial 1992; 8: 410-5.

15. Geary DF, Harvey EA, MacMillan JH, Goodman Y, Scott M, Balfe WJ. The peritoneal equilibration test in children. Kidney Int 1992; 42: 102-5.

16. Hanna JD, Foreman JW, Gehr TWB, Chan JCM, Wolfrum J, Ruddley J. The peritoneal equilibration test in children. Pediatr Nephrol 1993; 7:731 -4. 